\title{
DUAL INTEGRAL EQUATIONS INVOLVING WEBER-ORR TRANSFORMS
}

C. NASIM

\author{
Department of Mathematics \& Statistics \\ University of Calgary \\ Calgary, Alberta, Canada \\ T2N 1N4
}

(Received August 22, 1989 and in revised form July 19, 1990)

ABSTRACT. In this paper we consider dual integral equations involving inverse Weber-Orr transforms of the type $\mathrm{W}_{\nu-\kappa, \nu}^{-1}[;], \kappa=0,1, \ldots$. A general solution is established using elementary methods. Many known results are derived as special cases.

KEY WORDS AND PHRASES. Weber-Orr transforms, Bessel functions of the first, the second kind, and the third kind, Fredholm type integrals, Erdelyi-Kober integral operators. 1980 AMS SUBJECT CLASSIFICATION CODE. 44A20.

\section{INTRODUCTION.}

1. Dual integral equations involving inverse Weber-Orr transforms $W_{\mu, \nu}^{-1}[;]$, arise in the discussion of boundary value problems in elasticity and torsion. A few special cases have been considered, notabally, dual integral equations involving $w_{\nu, \nu}^{-1}[i]$ and $w_{0,1}^{-1}[;]$, by various authors, [1,2]. But there does not seem to have been given a full account of dual integral equations of general type in the literature. In this paper we shall consider equations involving associated Weber-Orr transforms of the type

$$
\begin{aligned}
& \mathrm{W}_{\nu-\kappa, \nu}^{-1}\left[\xi^{-2 \alpha} \psi(\xi) ; \rho\right]=\mathrm{f}_{1}(\rho), \quad a \leq \rho \leq \mathrm{c} \\
& \mathrm{W}_{\nu-\kappa, \nu}^{-1}\left[\xi^{-2 \beta} \psi(\xi) ; \rho\right]=-\mathrm{f}_{2}(\rho), \quad \mathrm{c}<\rho<\infty
\end{aligned}
$$

where $\kappa=0,1,2, \ldots, \nu>-1$ and $\psi$ an unknown function. Since associated Weber-Orr transforms are of general order $(\nu-\kappa, \nu)$, therefore most of the known results may be derived as special cases of our results. We may also point out, that special cases of (1.2) can arise when solving Beltrami-Michel equations in case of torsion, [2]. 
To solve the system (1.1), first we decompose it into two sets of dual integral equations when $f_{1}=0$ and $f_{2}=0$ respectively. The solution of the system can then be obtained by adding the solutions of the two sets. The method we use to find the solutions is similar to the one used in [3]. An important aspect of this method is to establish a suitable form of the unknown function $\psi$ in terms of an undetermined function, say g. The problem is then reduced to finding $\mathrm{g}$. In order that $\psi$ satisfies both the integral equations of the set, we find that $g$ satisfies a single integral equation of Fredholm type of the second kind. The value of $g$ can then be estimated, thus establishing the solution $\psi$ of the set.

For convenience, we shall state below some results and definitions for future reference. All those results are either known or can easily be derived by using well-known properties of the Bessel functions.

DEFINITION 1. We define,

$$
\begin{aligned}
& \mathrm{W}_{\mu, \nu}[\mathrm{f}(\rho) ; \xi]=\int_{\mathrm{a}}^{\infty} \Re_{\mu, \nu}(\xi ; \rho, \mathrm{a}) \rho \mathrm{f}(\rho) \mathrm{d} \rho \\
& \mathrm{W}_{\mu, \nu}^{-1}[\hat{\mathrm{f}}(\xi) ; \rho]=\int_{0}^{\infty} \frac{\Re_{\mu, \nu}(\xi ; \rho, \mathrm{a})}{\mathrm{J}_{\nu}^{2}(\xi \mathrm{a})+\mathrm{Y}_{\nu}^{2}(\xi \mathrm{a})} \xi \hat{\mathrm{f}}(\xi) \mathrm{d} \xi
\end{aligned}
$$

where

$$
\Re_{\mu, \nu}(\xi ; \rho, \mathrm{a})=\mathrm{J}_{\mu}(\xi \rho) \mathrm{Y}_{\nu}(\xi \mathrm{a})-\mathrm{Y}_{\mu}(\xi \rho) \mathrm{J}_{\nu}(\xi \mathrm{a}),
$$

$\mathrm{J}_{\mu}$ and $\mathrm{Y}_{\mu}$ being the usual Bessel functions. We shall call $\mathrm{W}_{\mu, \nu}[;]$ and $\mathrm{W}_{\mu, \nu}^{-1}[;]$ the associated Weber-Orr transform and inverse transform of order $(\mu, \nu)$, respectively.

DEFINITION 2, [4]. The Erdyli-Kober operators

$$
\mathscr{F}_{\eta, \alpha}[\mathrm{g}(\mathrm{x})]=\frac{2}{\Gamma(\alpha)} \mathrm{x}^{2 \eta} \int_{\mathrm{x}}^{\infty}\left(\mathrm{u}^{2}-\mathrm{x}^{2}\right)^{\alpha-1} \mathrm{u}^{1-2 \alpha-2 \eta} \mathrm{g}(\mathrm{u}) \mathrm{du}, \quad \text { if } \alpha>0
$$

and

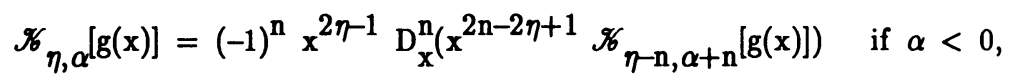

where

$$
\mathrm{D}_{\mathrm{x}}=\frac{1}{2} \frac{\mathrm{d}}{\mathrm{dx}} \frac{1}{\mathrm{x}} \text { and } 0 \leq \alpha+\mathrm{n}<1
$$

Also,

$$
\mathscr{\mathscr { K }}_{\eta, \alpha}^{1}=\mathscr{\mathscr { C }}_{\eta+\alpha,-\alpha}
$$

LEMMA 1. If $0<\kappa<\frac{1}{2} \nu+\frac{3}{4}, \kappa=0,1,2, \ldots$ and $\mathrm{x}^{\kappa+\frac{1}{2}} \mathrm{f}(\mathrm{x})$ is summable in the infinite interval $(a, \infty)$, then

$$
\hat{f}(\xi)=W_{\nu-\kappa, \nu}[f(\rho) ; \xi], \quad f(\rho)=W_{\nu-\kappa, \nu}^{-1}[\hat{f}(\xi) ; \rho] .
$$

This result has been proved by the author elsewhere. 
LEMMA 2, [1]. For $\alpha<0$, we define,

$$
\begin{aligned}
\mathscr{H}_{\nu, \eta, \mu, \alpha}(\rho, \mathrm{t})=\int_{0}^{\infty} \frac{\Re_{\eta, \nu}(\xi ; \rho, \mathrm{a}) \mathscr{R}_{\mu, \nu}(\xi ; \mathrm{t}, \mathrm{a})}{\mathrm{J}_{\nu}^{2}(\xi \mathrm{a})+\mathrm{Y}_{\nu}^{2}(\xi \mathrm{a})} \xi^{1+\alpha} \mathrm{d} \xi \\
=\int_{0}^{\infty} \mathrm{J}_{\eta}(\xi \rho) \mathrm{J}_{\mu}(\xi \mathrm{t}) \xi^{1+\alpha} \mathrm{d} \xi \\
-\frac{2}{\pi} \cos \frac{\pi}{2}(2 \nu-\eta-\mu+\alpha+1) \int_{0}^{\infty} \frac{\mathrm{I}_{\nu}(\mathrm{ay})}{\mathrm{K}_{\nu}(\mathrm{ay})} \mathrm{K}_{\eta}(\rho \mathrm{y}) \mathrm{K}_{\mu}(\mathrm{ty}) \mathrm{y}^{1+\alpha} \mathrm{dy}
\end{aligned}
$$

$\mathrm{K}_{\nu}$ being Bessel function of the third kind.

LEMMA 3, [5]. For $\nu>\mu>-1$,

$$
\int_{0}^{\infty} \mathrm{J}_{\mu}(\mathrm{au}) \mathrm{J}_{\nu}(\mathrm{bu}) \mathrm{u}^{\mu-\nu+1} \mathrm{du}=\left\{\begin{array}{cl}
0, & 0<\mathrm{b}<\mathrm{a} \\
\frac{2^{\mu-\nu+1} \mathrm{a}^{\mu}\left(\mathrm{b}^{2}-\mathrm{a}^{2}\right)^{\nu-\mu-1}}{\Gamma(\nu-\mu) \mathrm{b}^{\nu}}, & \mathrm{a}<\mathrm{b}<\infty .
\end{array}\right.
$$

LEMMA 4, [5]. For $\beta>0$,

$$
\int_{\mathrm{y}}^{\infty}\left(\mathrm{u}^{2}-\mathrm{y}^{2}\right)^{\beta-1} \mathrm{u}^{1-\eta} \mathrm{K}_{\eta}(\xi \mathrm{u}) \mathrm{du}=2^{\beta-1} \Gamma(\beta) \mathrm{y}^{\beta-\eta} \xi^{-\beta_{\mathrm{K}}}{ }_{\eta-\beta}(\xi \mathrm{y}) .
$$

LEMMA 5. For $0<\beta<\frac{1}{2} \eta+\frac{3}{4}$,

$$
\int_{\mathbf{y}}^{\infty}\left(\mathrm{u}^{2}-\mathrm{y}^{2}\right)^{\beta-1} \mathrm{u}^{1-\eta} \Re_{\eta, \nu}(\xi ; \mathrm{u}, \mathrm{a}) \mathrm{du}=2^{\beta-1} \Gamma(\beta) \mathrm{y}^{\beta-\eta} \xi^{-\beta} \Re_{\eta-\beta, \nu}(\xi ; \mathrm{y}, \mathrm{a}) .
$$

LEMMA 6. For $0<\beta<\frac{1}{2} \eta+\frac{3}{4}$,

$$
\int_{y}^{\infty^{\alpha}}\left(u^{2}-y^{2}\right)^{\beta-1} u^{1-\eta} \mathscr{H}_{\nu, \eta, \mu, \alpha}(u, t) d t=2^{\beta-1} \Gamma(\beta) y^{\beta-\eta} \mathscr{H}_{\nu, \eta-\beta, \mu, \alpha-\beta}(y, t) .
$$

2. We consider the dual integral equations

$$
\begin{aligned}
& \mathrm{W}_{\nu-\kappa, \nu}^{-1}\left[\xi^{-2 \alpha} \psi(\xi) ; \rho\right]=0 \quad, \quad \mathrm{a} \leq \rho \leq \mathrm{c} \\
& \mathrm{W}_{\nu-\kappa, \nu}^{-1}\left[\xi^{-2 \beta} \psi(\xi) ; \rho\right]=-\mathrm{f}_{2}(\rho), \quad \mathrm{c}<\rho<\infty .
\end{aligned}
$$

CASE (a). Let $-1<\alpha-\beta<0$.

The equation (2.1) is satisfied if we take

$$
\mathrm{W}_{\nu-\kappa, \nu}^{-1}\left[\xi^{-2 \alpha} \psi(\xi) ; \rho\right]=\mathrm{H}(\rho-c) \mathrm{G}(\rho)
$$

where $H$ is the Heaviside function and $G$, is yet unspecified. Then due to lemma 1 , for $0<\kappa<\frac{1}{2} \nu+\frac{3}{4}$,

$$
\xi^{-2 \alpha} \psi(\xi)=\mathrm{W}_{\nu-\kappa, \nu}[\mathrm{H}(\rho-\mathrm{c}) \mathrm{G}(\rho) ; \xi]=\int_{\mathrm{c}}^{\infty} \Re_{\nu-\kappa, \nu}(\xi ; \rho, \mathrm{a}) \rho \mathrm{G}(\rho) \mathrm{d} \rho
$$


Now using the representation for $\Re_{\nu-\kappa, \nu}$ from lemma 5 , and rearranging, we obtain for $-1<\alpha-\beta<0$ and $\beta-\alpha<\nu-\kappa+\frac{3}{2}$

$$
\begin{aligned}
& \psi(\xi)=\frac{2^{\alpha-\beta+1}}{\Gamma(\beta-\alpha)} \xi^{\beta+\alpha} \int_{\mathrm{c}}^{\infty} \rho^{1+\nu-\kappa} \mathrm{G}(\rho) \mathrm{d} \rho \int_{\rho}^{\infty}\left(\mathrm{t}^{2}-\mathrm{p}^{2}\right)^{\beta-\alpha-1} \mathrm{t}^{\kappa-\nu+\alpha-\beta+1} . \\
& \cdot \Re_{\nu-\mathrm{k}+\beta-\alpha, \nu}(\xi ; \mathrm{t}, \mathrm{a}) \mathrm{dt} \\
& =\frac{2^{\alpha-\beta+1}}{\Gamma(\beta-\alpha)} \xi^{\beta+\alpha} \int_{\mathrm{c}}^{\infty} \mathrm{t}^{\kappa-\nu+\alpha-\beta+1} \Re_{\nu-\kappa+\beta-\alpha, \nu}(\xi ; \mathrm{t}, \mathrm{a}) \mathrm{dt} \int_{\mathrm{c}}^{\mathrm{t}}\left(\mathrm{t}^{2}-\rho^{2}\right)^{\beta-\alpha-1} . \\
& \cdot \rho^{1+\nu-\kappa} \mathrm{G}(\rho) \mathrm{d} \rho .
\end{aligned}
$$

If we let

$$
g(t)=\frac{2^{\alpha-\beta+1}}{\Gamma(\beta-\alpha)} \mathrm{t}^{\kappa-\nu+\alpha-\beta+1} \int_{\mathrm{c}}^{\mathrm{t}}\left(\mathrm{t}^{2}-\rho^{2}\right)^{\beta-\alpha-1} \rho^{1+\nu-\kappa} \mathrm{G}(\rho) \mathrm{d} \rho
$$

then

$$
\psi(\xi)=\xi^{\beta+\alpha} \int_{\mathrm{c}}^{\infty} \Re_{\nu-\kappa+\beta-\alpha, \nu}(\xi ; \mathrm{t}, \mathrm{a}) \mathrm{g}(\mathrm{t}) \mathrm{dt}
$$

which gives us a suitable expression for $\psi$ in terms of as yet to be determined function $\mathrm{g}$. Now for $\rho>c$, from (2.2),

$$
\begin{gathered}
-\mathrm{f}_{2}(\rho)=\mathrm{W}_{\nu-\kappa, \nu}^{-1}\left[\xi^{-2 \beta} \psi(\xi) ; \rho\right] \\
=\int_{0}^{\infty} \frac{\Re{ }_{\nu-\kappa, \nu}(\xi ; \rho, \mathrm{a})}{\mathrm{J}_{\nu}^{2}(\xi \mathrm{a})+\mathrm{Y}_{\nu}^{2}(\xi \mathrm{a})} \xi^{1-2 \beta} \psi(\xi) \mathrm{d} \xi .
\end{gathered}
$$

Substituting the value of $\psi$ from (2.4), and changing the order of integration, we have,

$$
-\mathrm{f}_{2}(\rho)=\int_{\mathrm{c}}^{\infty} \mathrm{g}(\mathrm{t}) \not{H} \nu, \nu-\kappa, \nu-\kappa+\beta-\alpha, \alpha-\beta(\rho, \mathrm{t}) \mathrm{dt} .
$$

Using the results of lemmas 2 and 3 , for $c<\rho<t<\omega$, we may write $\mathscr{H}_{\nu, \nu-\kappa, \nu-\kappa+\beta-\alpha, \alpha-\beta}(\rho, \mathrm{t})=\frac{2^{1+\alpha-\beta}}{\Gamma(\beta-\alpha)} \rho^{\nu-\kappa} \mathrm{t}^{\kappa-\nu+\alpha-\beta}\left(\mathrm{t}^{2}-\rho^{2}\right)^{\beta-\alpha-1} \mathrm{H}(\mathrm{t}-\rho)+\phi(\rho, \mathrm{t})$, where

$$
\phi(\rho, \mathrm{t})=\frac{2}{\pi} \sin \pi(\kappa+\alpha-\beta) \int_{0}^{\infty} \frac{\mathrm{I}_{\nu}(\mathrm{ay})}{\mathrm{K}_{\nu}(\mathrm{ay})} \mathrm{K}_{\nu-\kappa}(\rho \mathrm{y}) \mathrm{K}_{\nu-\kappa+\beta-\alpha}(\mathrm{ty}) \mathrm{y}^{1+\alpha-\beta} \mathrm{dy},
$$

and $\kappa+1>\beta-\alpha$.

Hence (2.5) gives

$$
-\mathrm{f}_{2}(\rho)=\frac{2^{1+\alpha-\beta}}{\Gamma(\beta-\alpha)} \rho^{\nu-\kappa} \int_{\rho}^{\infty} \mathrm{t}^{\kappa-\nu+\alpha-\beta}\left(\mathrm{t}^{2}-\rho^{2}\right)^{\beta-\alpha-1} \mathrm{~g}(\mathrm{t}) \mathrm{dt}+\int_{\mathrm{c}}^{\infty} \mathrm{g}(\mathrm{t}) \phi(\rho, \mathrm{t}) \mathrm{dt} .
$$

Now multiply both sides by $2^{q-\alpha} \rho^{1+\alpha-\beta}$ and make use of the Erdyli-Kober operator $\mathscr{F}_{\eta, \alpha}$, then the last equation, on rearranging, gives, 
DUAL INTEGRAL EQUATIONS

167

$$
\begin{gathered}
\mathscr{F}_{\frac{1}{2}(\nu-\kappa+\alpha-\beta+1), \beta-\alpha}[\mathrm{g}(\rho)]=-2^{\beta-\alpha} \rho^{1+\alpha-\beta} \int_{\mathrm{c}}^{\infty} \mathrm{g}(\mathrm{t}) \phi(\rho, \mathrm{t}) \mathrm{dt}-2^{\beta-\alpha} \rho^{1+\alpha-\beta} \mathrm{f}_{2}(\rho) \\
=-\mathrm{G}(\rho)-\mathrm{F}(\rho), \text { say. }
\end{gathered}
$$

Hence for $-1<\alpha-\beta<0$,

$$
\begin{aligned}
\mathrm{g}(\rho) & =-\mathscr{K}_{\frac{1}{2}}^{-1}(\nu-\kappa+\alpha-\beta+1), \beta-\alpha \\
& =-\mathscr{F}_{\frac{1}{2}}(\nu-\kappa+\beta-\alpha+1), \alpha-\beta[\mathrm{G}(\rho)+\mathrm{F}(\rho)] \\
& =\frac{1}{2} \rho^{\nu-\kappa+\beta-\alpha} \frac{\mathrm{d}}{\mathrm{d} \rho}\left[\rho^{\kappa-\nu+\alpha-\beta+1} \mathscr{K}_{\frac{1}{2}(\nu-\kappa+\beta-\alpha+1), 1+\alpha-\beta}[\mathrm{G}(\rho)+\mathrm{F}(\rho)]\right] \\
& =\rho^{\nu-\kappa+\beta-\alpha} \mathrm{d} \\
\Gamma(1+\alpha-\beta) & \frac{\mathrm{d} \rho}{\mathrm{d} \rho} \int_{\rho}^{\infty}\left(\mathrm{u}^{2}-\rho^{2}\right)^{\alpha-\beta} \mathrm{u}^{\kappa-\nu+\beta-\alpha}[\mathrm{G}(\mathrm{u})+\mathrm{F}(\mathrm{u})] \mathrm{du} .
\end{aligned}
$$

Substituting the values of $F$ and $G$ in the above equation, we obtain,

$$
\begin{aligned}
& \mathrm{g}(\rho)=\frac{2^{\beta-\alpha}}{\Gamma(1+\alpha-\beta)} \rho^{\nu-\kappa+\beta-\alpha} \frac{\mathrm{d}}{\mathrm{d} \rho}\left[\int_{\mathrm{c}}^{\infty} \mathrm{g}(\mathrm{t}) \mathrm{dt} \int_{\rho}^{\infty}\left(\mathrm{u}^{2}-\rho^{2}\right)^{\alpha-\beta} \mathrm{u}^{1+\kappa-\nu} \phi(\mathrm{u}, \mathrm{t}) \mathrm{du}\right] \\
& \quad+\frac{{ }^{\beta-\alpha}}{\Gamma(1+\alpha-\beta)} \rho^{\nu-\kappa+\beta-\alpha} \frac{\mathrm{d}}{\mathrm{d} \rho}\left[\int_{\rho}^{\infty}\left(\mathrm{u}^{2}-\rho^{2}\right)^{\alpha-\beta} \mathrm{u}^{1+\kappa-\nu} \mathrm{f}_{2}(\mathrm{u}) \mathrm{du}\right] \\
& =\mathrm{I}_{1}+\mathrm{I}_{2} \text {, say. }
\end{aligned}
$$

On substituting for $\phi$ in $I_{1}$, we have

$$
\begin{gathered}
\mathrm{I}_{1}=\frac{2^{\beta-\alpha+1}}{\pi \Gamma(1+\alpha-\beta)} \rho^{\nu-\kappa+\beta-\alpha} \cdot \sin \pi(\kappa+\alpha-\beta) \frac{\mathrm{d}}{\mathrm{d} \rho}\left[\int_{\mathrm{c}}^{\infty} \mathrm{g}(\mathrm{t}) \mathrm{dt} \int_{\rho}^{\infty}\left(\mathrm{u}^{2}-\rho^{2}\right)^{\alpha-\beta} \mathrm{u}^{1+\kappa-\nu} \mathrm{du} .\right. \\
\cdot \int_{0}^{\infty} \frac{\mathrm{I}_{\nu}(\mathrm{ay})}{\mathrm{K}_{\nu}(\mathrm{ay})} \mathrm{K}_{\nu-\kappa}(\mathrm{uy}) \mathrm{K}_{\nu-\kappa+\beta-\alpha}(\mathrm{ty}) \mathrm{y}^{1+\alpha-\beta} \mathrm{dy} .
\end{gathered}
$$

Now, first evaluate the u-integral, using the result of lemma 4 , then apply the operator $\frac{d}{d \rho}$ to obtain

$$
\mathrm{I}_{1}=\frac{-2 \rho}{\pi} \sin \pi(\kappa+\alpha-\beta) \int_{\mathrm{c}}^{\infty} \mathrm{g}(\mathrm{t}) \mathrm{dt} \int_{0}^{\infty} \frac{\mathrm{I}_{\nu}(\mathrm{ay})}{\mathrm{K}_{\nu}(\text { ayr })} \mathrm{K}_{\nu-\kappa+\beta-\alpha}(\rho \mathrm{y}) \mathrm{K}_{\nu-\kappa+\beta-\alpha}(\mathrm{ty}) \mathrm{y} \mathrm{dy} .
$$

Hence, from (2.8), for $-1<\alpha-\beta<0$,

$$
\begin{aligned}
\mathrm{g}(\rho) & =\frac{-2 \rho}{\pi} \sin \pi(\kappa+\alpha-\beta) \int_{\mathrm{c}}^{\infty} \mathrm{g}(\mathrm{t}) \mathrm{dt} \int_{0}^{\infty} \frac{\mathrm{I}_{\nu}(\mathrm{ay})}{\mathrm{K}_{\nu}(\mathrm{ay})} \mathrm{K}_{\nu-\kappa+\beta-\alpha}(\rho \mathrm{y}) \mathrm{K}_{\nu-\kappa+\beta-\alpha}(\mathrm{ty}) \mathrm{y} \mathrm{dy} \\
& +\frac{2^{\beta-\alpha}}{\Gamma(1+\alpha-\beta)} \rho^{\nu-\kappa+\beta-\alpha} \frac{\mathrm{d}}{\mathrm{d} \rho}\left[\int_{\rho}^{\infty}\left(\mathrm{u}^{2}-\rho^{2}\right)^{\alpha-\beta} \mathrm{u}^{1+\kappa-\nu} \mathrm{f}_{2}(\mathrm{u}) \mathrm{du}\right]
\end{aligned}
$$

where (i) $0<\kappa<\frac{1}{2} \nu+\frac{3}{4}$, (ii) $\beta-\alpha<\nu-\kappa+\frac{3}{2}$, (iii) $\beta-\alpha<\kappa+1$. 
This is a linear integral equation of Fredholm type of the second kind, from where $\mathrm{g}$ can be evaluated, thus giving us a solution $\psi$ of the dual integral equations (2.1), (2.2). The conditions of validity of the Fredholm equation (2.9) is obviously that the integral defining the kernel

$$
\int_{0}^{\infty} \frac{\mathrm{I}{ }_{\nu}(\mathrm{ay})}{\mathrm{K}_{\nu}(\mathrm{ay})} \underset{\nu-\mathrm{K}+\beta-\alpha}{\mathrm{K}} \stackrel{(\mathrm{py}) \mathrm{K}}{\nu-\mathrm{K}+\beta-\alpha} \underset{ }{(\mathrm{ty}) \mathrm{ydy}}
$$

converges. The convergence is guaranteed when $\beta-\alpha<\mathrm{k}+1$ and $2 \mathrm{a}<\rho+\mathrm{t}$, since $0<\mathrm{a}<\rho$ and $\mathrm{a}<\mathrm{c}<\mathrm{t}$. Next we shall show that this solution is also valid when $0<\alpha-\beta<1$, but the derivation will be slightly different.

CASE (b). Let $0<\alpha-\beta<1$.

The equation (2.1) is satisfied if we take

$$
\mathrm{W}_{\nu-\kappa, \nu}^{-1}\left[\xi^{-2 \alpha} \psi(\xi) ; \rho\right]=\mathrm{H}(\rho-c) \mathrm{G}(\rho)
$$

where $\mathrm{G}(\mathrm{c})=0$ and $\sqrt{\rho} \mathrm{G}(\rho) \rightarrow 0$ as $\rho \rightarrow \infty$. Then for $0<\kappa<\frac{1}{2} \nu+\frac{3}{4}$,

$$
\begin{aligned}
\xi^{-2 \alpha} \psi(\xi) & =\mathrm{W}_{\nu-\kappa, \nu}[\mathrm{H}(\rho-\mathrm{c}) \mathrm{G}(\rho) ; \xi] \\
& =\int_{\mathrm{c}}^{\infty} \Re_{\nu-\kappa, \nu}(\xi ; \rho, \mathrm{a}) \rho \mathrm{G}(\rho) \mathrm{d} \rho \\
& =\frac{1}{\xi} \int_{\mathrm{c}}^{\infty} \frac{\mathrm{d}}{\mathrm{d} \rho}\left(\rho^{\nu-\kappa} \mathrm{G}(\rho)\right) \rho^{\kappa-\nu+1} \Re_{\nu-\kappa-1, \nu}(\xi ; \rho, \mathrm{a}) \mathrm{d} \rho
\end{aligned}
$$

by integrating by parts. Now using the representation for $\Re_{\nu-\kappa-1, \nu}(\xi ; \rho, \mathrm{a})$ from lemma 5 and changing the order of integration, we obtain for $0<\alpha-\beta<1$ and $\beta-\alpha<\nu-\kappa-\frac{1}{2}$

$$
\psi(\xi)=\xi^{\alpha+\beta} \int_{\mathrm{c}}^{\infty} \Re_{\nu-\kappa+\beta-\alpha, \nu}(\xi ; \mathrm{t}, \mathrm{a}) \mathrm{g}(\mathrm{t}) \mathrm{dt}
$$

where

$$
g(t)=\frac{2^{\alpha-\beta}}{\Gamma(1+\beta-\alpha)} \mathrm{t}^{\kappa-\nu-\beta+\alpha+1} \int_{\mathrm{c}}^{\mathrm{t}} \frac{\mathrm{d}}{\mathrm{d} \rho}\left(\rho^{\nu-\kappa} \mathrm{G}(\rho)\right)\left(\mathrm{t}^{2}-\rho^{2}\right)^{\beta-\alpha} \mathrm{d} \rho
$$

Notice that the representation for $\psi(\xi)$ in (2.11) is identical to the one given in (2.4). If we take $\rho>c$, then (2.2) gives

$$
\begin{aligned}
-\mathrm{f}_{2}(\rho) & =\mathrm{w}_{\nu-\kappa, \nu}^{-1}\left[\xi^{-2 \beta} \psi(\xi) ; \rho\right] \\
& =\int_{0}^{\infty} \frac{\Re_{\nu-\kappa, \nu}(\xi ; \rho, \mathrm{a})}{\mathrm{J}_{\nu}^{2}(\xi \mathrm{a})+\mathrm{Y}_{\nu}^{2}(\xi \mathrm{a})} \xi^{1-2 \beta} \psi(\xi) \mathrm{d} \xi
\end{aligned}
$$

or,

$$
\rho^{\kappa-\nu+1} \mathrm{f}_{2}(\rho)=\frac{\mathrm{d}}{\mathrm{d} \rho}\left[\rho^{\kappa-\nu+1} \int_{\mathrm{c}}^{\infty} \frac{\Re_{\nu-\kappa-1, \nu}(\xi ; \rho, \mathrm{a})}{\mathrm{J}_{\nu}^{2}(\xi \mathrm{a})+\mathrm{Y}_{\nu}^{2}(\xi \mathrm{a})} \xi^{-2 \beta} \psi(\xi) \mathrm{d} \xi\right] .
$$


Now if we replace $\psi$ by its value given by the equation (2.11), and rearrange, then

$$
\rho^{\kappa-\nu+1} \mathrm{f}_{2}(\rho)=\frac{\mathrm{d}}{\mathrm{d} \rho}\left[\rho^{\kappa-\nu+1} \int_{\mathrm{c}}^{\infty} \not{ }_{\nu, \nu-\kappa-1, \nu-\kappa+\beta-\alpha, \alpha-\beta-1}(\rho, \mathrm{t}) \mathrm{g}(\mathrm{t}) \mathrm{dt}\right]
$$

Making use of lemmas 2 and 3, and simplifying, we obtain for $\nu-\kappa>0$,

$$
\begin{aligned}
\rho^{\kappa-\nu+1} \mathrm{f}_{2}(\rho) & =\frac{2^{\alpha-\beta}}{\Gamma(1+\beta-\alpha)} \frac{\mathrm{d}}{\mathrm{d} \rho} \int_{\rho}^{\infty}\left(\mathrm{t}^{2}-\rho^{2}\right)^{\beta-\alpha} \mathrm{t}^{\kappa-\nu+\alpha-\beta} \mathrm{g}(\mathrm{t}) \mathrm{dt} \\
& +\frac{2}{\pi} \sin \pi(\kappa+\alpha-\beta) \frac{\mathrm{d}}{\mathrm{d} \rho}\left[\rho^{\kappa-\nu+1} \int_{\mathrm{c}}^{\infty} \mathrm{g}(\mathrm{t}) \phi(\rho, \mathrm{t}) \mathrm{dt}\right]
\end{aligned}
$$

where

$$
\phi(\rho, \mathrm{t})=\int_{0}^{\infty} \frac{I_{\nu}(\mathrm{ay})}{K_{\nu}(\mathrm{ay})} K_{\nu-\kappa-1}(\rho \mathrm{y}) \mathrm{K}_{\nu-\kappa+\beta-\alpha}(\mathrm{ty}) \mathrm{y}^{\alpha-\beta} \mathrm{dy}
$$

Now using Erdyli-Kober operator $\mathscr{F}_{\eta, \alpha}$, the first term on the right-hand side of (2.12), gives

$$
\begin{array}{rl}
2^{\alpha-\beta-1} & \mathrm{~d}\left[\rho^{\kappa-\nu+\beta-\alpha+1} \mathscr{K}_{\frac{1}{2}(\nu-\kappa+\alpha-\beta-1), \beta-\alpha+1}[\lg (\rho)]\right] \\
\quad=-2^{\alpha-\beta} \rho^{\kappa-\nu+\beta-\alpha} \mathscr{K}_{\frac{1}{2}(\nu-\kappa+\alpha-\beta+1), \beta-\alpha}[\operatorname{g}(\rho)] .
\end{array}
$$

Hence from (2.12), we obtain

$$
\begin{aligned}
\mathscr{F}_{\frac{1}{2}(\nu-\kappa+\alpha-\beta+1), \beta-\alpha}[\mathrm{g}(\rho)] & =-2^{\beta-\alpha} \rho^{\alpha-\beta+1} \mathrm{f}_{2}(\rho)+\frac{2}{\pi}^{\beta-\alpha+1} \sin \pi(\kappa+\alpha-\beta) \\
& \cdot \rho^{\nu-\kappa+\alpha-\beta} \frac{\mathrm{d}}{\mathrm{d} \rho}\left[\rho^{\kappa-\nu+1} \int_{\mathrm{c}}^{\infty} \mathrm{g}(\mathrm{t}) \phi(\rho, \mathrm{t}) \mathrm{dt}\right] \\
& =\mathrm{F}(\rho)+\mathrm{G}(\rho), \text { say. }
\end{aligned}
$$

Therefore for $0<\alpha-\beta<1$,

$$
\begin{aligned}
& \mathrm{g}(\rho)=\mathscr{K}_{\frac{1}{2}(\nu-\kappa+\alpha-\beta+1), \beta-\alpha}^{-1}[\mathrm{~F}(\rho)+\mathrm{G}(\rho)] . \\
& =\mathscr{K}_{\frac{1}{2}}(\nu-\kappa+\beta-\alpha+1), \alpha-\beta[\mathrm{F}(\rho)+\mathrm{G}(\rho)] \\
& =\frac{2}{\Gamma(\alpha-\beta)} \rho^{\nu-\kappa+\beta-\alpha+1} \int_{\rho}^{\infty}\left(\mathrm{u}^{2}-\rho^{2}\right)^{\alpha-\beta-1} \mathrm{u}^{\kappa-\nu+\beta-\alpha}[\mathrm{F}(\mathrm{u})+\mathrm{G}(\mathrm{u})] \mathrm{du} \\
& =\frac{-2^{\beta-\alpha+1}}{\Gamma(\alpha-\beta)} \rho^{\nu-\kappa+\beta-\alpha+1} \int_{\rho}^{\infty}\left(u^{2}-\rho^{2}\right)^{\alpha-\beta-1} u^{\kappa-\nu+1} f_{2}(u) d u \\
& +\frac{2^{\beta-\alpha+2}}{\pi \Gamma(\alpha-\beta)} \sin \pi(\kappa+\alpha-\beta) \rho^{\nu-\kappa+\beta-\alpha+1} \int_{\rho}^{\infty}\left(u^{2}-\rho^{2}\right)^{\alpha-\beta-1} \frac{d}{d u}\left(u^{\kappa-\nu+1} .\right. \\
& \left.\int_{c}^{\infty} g(t) \phi(u, t) d t\right) d u \\
& =I_{1}+I_{2} \text {, say. }
\end{aligned}
$$


In $I_{2}$, first we substitute for $\phi(u, t)$ and apply the operator $\frac{d}{d u}$, then evaluate the u-integral using the result of lemma 4 , hence, finally, gives for $0<\alpha-\beta<1$,

$$
\begin{aligned}
& \mathrm{g}(\rho)=-\frac{2^{\beta-\alpha+1}}{\Gamma(\alpha-\beta)} \rho^{\nu-\kappa+\beta-\alpha+1} \int_{\rho}^{\infty}\left(\mathrm{u}^{2}-\rho^{2}\right)^{\alpha-\beta-1} \mathrm{u}^{\kappa-\nu+1} \mathrm{f}_{2}(\mathrm{u}) \mathrm{du} \\
& -\frac{2}{\pi} \sin \pi(\kappa+\alpha-\beta) \rho \int_{\mathrm{c}}^{\infty} \mathrm{g}(\mathrm{t}) \mathrm{dt} \int_{0}^{\infty} \frac{\mathrm{I}_{\nu}(\mathrm{ay})}{\mathrm{K}_{\nu}(\mathrm{ay})} \mathrm{K}_{\nu-\kappa+\beta-\alpha}(\rho \mathrm{y}) \mathrm{K}_{\nu-\kappa+\beta-\alpha}(\mathrm{ty}) \mathrm{ydy},
\end{aligned}
$$

where (i) $0<\kappa<\frac{1}{2} \nu+\frac{3}{4}, \quad$ (ii) $\beta-\alpha<\nu-\kappa-\frac{1}{2}, \quad$ (iii) $\beta-\alpha<\mathrm{L}+1$.

This is a linear integral equation of Fredholm type of the second kind and is identical to the one for the case $-1<\alpha-\beta<0$, given in (2.9).

Hence the solution $\psi$ for the dual integral equation (2.1) and (2.2) is given by

$$
\psi(\xi)=\xi^{\alpha+\beta} \int_{\mathrm{c}}^{\infty} \Re_{\nu-\kappa+\beta-\alpha, \nu}(\xi ; \mathrm{t}, \mathrm{a}) \mathrm{g}(\mathrm{t}) \mathrm{dt}
$$

where $\mathrm{g}$ satisfies the Fredholm equation (2.9) or (2.13) for $-1<\alpha-\beta<1$, $\alpha-\beta \neq 0$.

3. Next we consider the dual integral equations

$$
\begin{array}{ll}
\mathrm{W}_{\nu-\kappa, \nu}^{-1}\left[\xi^{-2 \alpha} \psi(\xi) ; \rho\right]=\mathrm{f}_{1}(\rho), & \mathrm{a} \leq \rho \leq \mathrm{c} \\
\mathrm{W}_{\nu-\kappa, \nu}^{-1}\left[\xi^{-2 \beta} \psi(\xi) ; \rho\right]=0, & \mathrm{c}<\rho<\infty
\end{array}
$$

Again we shall consider two cases when $-1<\alpha-\beta<0$ and $0<\alpha-\beta<1$ separately and arrive at a common solution of the system, although the derivation will be slightly different for the two cases.

CASE (a). Let $-1<\alpha-\beta<0$.

Equation (3.1) is satisfied if we take

$$
\mathrm{W}_{\nu-\kappa, \nu}^{-1}\left[\xi^{-2 \alpha} \psi(\xi) ; \rho\right]=\mathrm{f}_{1}(\rho) \mathrm{H}(\mathrm{c}-\rho)+\mathrm{G}(\rho) \mathrm{H}(\rho-\mathrm{c})
$$

where $H$ is the Heaviside function and $G$, and unspecified function. Then for $0<\kappa<\frac{1}{2} \nu+\frac{3}{4}$

$$
\begin{aligned}
& \xi^{-2 \alpha} \psi(\xi)=\mathrm{W}_{\nu-\kappa, \nu}\left[\mathrm{f}_{1}(\rho) \mathrm{H}(\mathrm{c}-\rho)+\mathrm{G}(\rho) \mathrm{H}(\rho-\mathrm{c}) ; \xi\right] \\
& \quad=\int_{\mathrm{a}}^{\mathrm{c}} \Re_{\nu-\kappa, \nu}(\xi ; \rho, \mathrm{a}) \rho \mathrm{f}_{1}(\rho) \mathrm{d} \rho+\int_{\mathrm{c}}^{\infty} \Re_{\nu-\kappa, \nu}(\xi ; \rho, \mathrm{a}) \rho \mathrm{G}(\rho) \mathrm{d} \rho
\end{aligned}
$$

the second integral in the right-hand side of (3.3) is identical to the one given in (2.3), where it was evaluated to eventually give

$$
\xi^{\beta-\alpha} \int_{\mathrm{c}}^{\infty} \Re_{\nu-\kappa+\beta-\alpha, \nu}(\xi ; \mathrm{t}, \mathrm{a}) \mathrm{g}(\mathrm{t}) \mathrm{dt},
$$


where $g$ is yet to be determined. Thus we have

$\psi(\xi)=\xi^{2 \alpha} \int_{\mathrm{a}}^{\mathrm{c}} \AA_{\nu-\kappa, \nu}(\xi ; \mathrm{t}, \mathrm{a}) \mathrm{t} \mathrm{f}_{1}(\mathrm{t}) \mathrm{dt}+\xi^{\alpha+\beta} \int_{\mathrm{c}}^{\infty} \Re_{\nu-\kappa+\beta-\alpha, \nu}(\xi ; \mathrm{t}, \mathrm{a}) \mathrm{g}(\mathrm{t}) \mathrm{dt}$

Now let $\rho>$ c, so that from (3.2),

$$
\begin{gathered}
0=\mathrm{W}_{\nu-\kappa, \nu}^{-1}\left[\xi^{-2 \beta} \psi(\xi) ; \rho\right] \\
=\int_{0}^{\infty} \frac{\Re_{\nu-\kappa, \nu}(\xi ; \rho, \mathrm{a})}{\mathrm{J}_{\nu}^{2}(\xi \mathrm{a})+\mathrm{Y}_{\nu}^{2}(\xi \mathrm{a})} \xi^{1-2 \beta} \psi(\xi) \mathrm{d} \xi
\end{gathered}
$$

In substituting the value of $\psi$ from (3.4) and changing the order of integration we obtain

$$
0=\int_{a}^{c} \mathrm{t} \mathscr{H}_{\nu, \nu-\kappa, \nu-\kappa, 2 \alpha-2 \beta}(\rho, \mathrm{t}) \mathrm{f}_{1}(\mathrm{t}) \mathrm{dt}+\int_{\mathrm{c}}^{\infty} \mathscr{\not}{ }_{\nu, \nu-\kappa, \nu-\kappa+\beta-\alpha, \alpha-\beta}(\rho, \mathrm{t}) \mathrm{g}(\mathrm{t}) \mathrm{dt},
$$

where $\mathscr{H}(\rho . t)$ is defined in lemma 2 .

Note that the second term on the right-hand side is identical to the term on the right-hand side of (2.5). Therefore using a similar analysis, we arrive at the following equation,

$$
\begin{aligned}
& \mathscr{\mathscr { F }}_{\frac{1}{2}(\nu-\kappa+\alpha-\beta+1), \beta-\alpha}[\mathrm{g}(\rho)]=-2^{\beta-\alpha} \rho^{\alpha-\beta+1} \int_{\mathrm{c}}^{\infty} \phi(\rho, \mathrm{t}) \mathrm{g}(\mathrm{t}) \mathrm{dt} \\
& \quad-2^{\beta-\alpha} \rho^{\alpha-\beta+1} \int_{\mathrm{a}}^{\mathrm{c}} \mathrm{t} \mathscr{H}_{\nu, \nu-\kappa, \nu-\kappa, 2 \alpha-2 \beta}(\rho, \mathrm{t}) \mathrm{f}_{1}(\mathrm{t}) \mathrm{dt} \\
& =-\mathrm{G}(\rho)-\mathrm{F}(\rho), \text { say, }
\end{aligned}
$$

where $\phi(\rho, t)$ is defined by (2.6). And eventually, as before in (2.8),

$$
\begin{aligned}
g(\rho) & =\frac{2^{\beta-\alpha}}{\Gamma(1+\alpha-\beta)} \rho^{\nu-\kappa+\beta-\alpha} \frac{\mathrm{d}}{\mathrm{d} \rho} \int_{\mathrm{c}}^{\infty} \mathrm{g}(\mathrm{t}) \mathrm{dt} \int_{\rho}^{\infty}\left(\mathrm{u}^{2}-\rho^{2}\right)^{\alpha-\beta} \mathrm{u}^{1+\kappa-\nu} \phi(\mathrm{u}, \mathrm{t}) \mathrm{du} \\
+ & \frac{{ }^{\beta-\alpha}}{\Gamma(1+\alpha-\beta)} \rho^{\nu-\kappa+\beta-\alpha} \frac{\mathrm{d}}{\mathrm{d} \rho} \int_{\rho}^{\infty}\left(\mathrm{u}^{2}-\rho^{2}\right)^{\alpha-\beta} \mathrm{u}^{1+\kappa-\nu} \mathrm{du} \int_{\mathrm{a}}^{\mathrm{c}} \mathrm{t} \\
& \cdot \mathscr{H}_{\nu, \nu-\kappa, \nu-\kappa, 2 \alpha-2 \beta}(\mathrm{u}, \mathrm{t}) \mathrm{f}_{1}(\mathrm{t}) \mathrm{dt} .
\end{aligned}
$$

Now we first carry out the $u$-integration in both the terms, using the results of lemmas 4 and 6 ; then apply the operator $\frac{d}{d \rho}$, to finally obtain

$$
\begin{aligned}
\mathrm{g}(\rho)= & -\frac{2}{\pi} \sin \pi(\kappa+\alpha-\beta) \rho \int_{\mathrm{c}}^{\infty} \mathrm{g}(\mathrm{t}) \mathrm{dt} \int_{0}^{\infty} \frac{\mathrm{I}_{\nu}(\mathrm{ay})}{\mathrm{K}_{\nu}(\mathrm{ay})} \mathrm{K}_{\nu-\kappa+\beta-\alpha}(\rho \mathrm{y}) \mathrm{K}_{\nu-\kappa+\beta-\alpha}(\mathrm{ty}) \mathrm{y} \mathrm{dy} \\
& -\rho \int_{\mathrm{a}}^{\mathrm{c}} \mathrm{t} \mathscr{\mathscr { H }}{ }_{\nu, \nu-\kappa+\beta-\alpha, \nu-\kappa, \alpha-\beta}(\rho, \mathrm{t}) \mathrm{f}_{1}(\mathrm{t}) \mathrm{dt}
\end{aligned}
$$

This is a linear integral equation of Fredholm type of the second kind, from where $g$ can 
be evaluated, thus giving us the solution $\psi$, defined by (3.4), of the dual integral equations (3.1) and (3.2), for $-1<\alpha-\beta<0,0<\kappa<\frac{1}{2} \nu+\frac{3}{4}, \beta-\alpha<\nu-\kappa+\frac{3}{2}$ and $\beta-\alpha<\mathrm{K}+1$.

CASE (b). Let $0<\alpha-\beta<1$.

The equation (3.1) is satisfied if we take, as before,

$$
\mathrm{W}_{\nu-\kappa, \nu}^{-1}\left[\xi^{-2 \alpha} \psi(\xi) ; \rho\right]=\mathrm{f}_{1}(\rho) \mathrm{H}(\mathrm{c}-\rho)+\mathrm{G}(\rho) \mathrm{H}(\rho-\mathrm{c})
$$

where

$$
\mathrm{G}(\mathrm{c})=0 \text { and } \sqrt{\rho} \mathrm{G}(\rho) \rightarrow 0 \text { as } \rho \rightarrow \infty
$$

Then

$$
\xi^{-2 \alpha} \psi(\xi)=\int_{\mathrm{a}}^{\mathrm{c}} \Re_{\nu-\kappa, \nu}(\xi ; \rho, \mathrm{a}) \rho \mathrm{f}_{1}(\rho) \mathrm{d} \rho+\int_{\mathrm{c}}^{\mathrm{w}} \Re_{\nu-\kappa, \nu}(\xi ; \rho, \mathrm{a}) \rho \mathrm{G}(\rho) \mathrm{d} \rho .
$$

The second term on the right-hand side is identical to the one on the right-hand side of (2.10) and by a similar analysis, we eventually obtain,

$$
\psi(\xi)=\xi^{2 \alpha} \int_{\mathrm{a}}^{\mathrm{c}} \Re_{\nu-\kappa, \nu}(\xi ; \rho, \mathrm{a}) \rho \mathrm{f}_{1}(\rho) \mathrm{d} \rho+\xi^{\alpha+\beta} \int_{\mathrm{c}}^{\infty} \Re_{\nu-\kappa+\beta-\alpha, \nu}(\xi ; \mathrm{t}, \mathrm{a}) \mathrm{g}(\mathrm{t}) \mathrm{dt}
$$

This gives us representation for $\psi$, where $\mathrm{g}$ is yet an undetermined function. Note that representation for $\psi$ is identical to the one for the case $-1<\alpha-\beta<0$ given in (3.4). Now for $\mathrm{c}<\rho<\infty$, from (3.2),

$$
\begin{aligned}
0=\mathrm{W}_{\nu-\kappa, \nu}^{-1} & {\left[\xi^{-2 \beta} \psi(\xi) ; \rho\right] } \\
& =\int_{0}^{\infty} \frac{\Re_{\nu-\kappa, \nu}(\xi ; \rho, \mathrm{a})}{\mathrm{J}_{\nu}^{2}(\xi \mathrm{a})+\mathrm{Y}_{\nu}^{2}(\xi \mathrm{a})} \xi^{1-2 \beta} \psi(\xi) \mathrm{d} \xi \\
& =\frac{\mathrm{d}}{\mathrm{d} \rho}\left[\rho^{\kappa-\nu+1} \int_{0}^{\infty} \frac{\Re_{\nu-\kappa-1, \nu}(\xi ; \rho, \mathrm{a})}{\mathrm{J}_{\nu}^{2}(\xi \mathrm{a})+\mathrm{Y}_{\nu}^{2}(\xi \mathrm{a})} \xi^{-2 \beta} \psi(\xi) \mathrm{d} \xi\right]
\end{aligned}
$$

f on replacing $\psi$ by the expression given in (3.6), and simplifying, we obtain

$$
\begin{aligned}
& 0=-\rho^{\kappa-\nu+1} \int_{0}^{\infty} \frac{\Re_{\nu-\mathrm{k}, \nu}(\xi ; \rho, \mathrm{a})}{\mathrm{J}_{\nu}^{2}(\xi \mathrm{a})+\mathrm{Y}_{\nu}^{2}(\xi \mathrm{a})} \xi^{2 \alpha-2 \beta+1} \mathrm{~d} \xi \int_{\mathrm{a}}^{\mathrm{c}} \Re_{\nu-\kappa, \nu}(\xi ; \mathrm{t}, \mathrm{a}) \mathrm{tf} \mathrm{f}_{1}(\mathrm{t}) \mathrm{dt}
\end{aligned}
$$

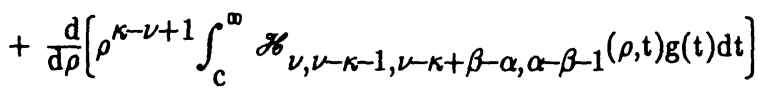

$$
\begin{aligned}
& =-2^{\alpha-\beta} \rho^{\kappa-\nu} \mathrm{F}(\mathrm{e})^{+\beta-\alpha}+\frac{2^{\alpha-\beta}}{\Gamma(\beta-\alpha+1)} \frac{\mathrm{d}}{\mathrm{d} \rho} \int_{\rho}^{\infty}\left(\mathrm{t}^{2}-\rho^{2}\right)^{\beta-\alpha} \mathrm{t}^{\kappa-\nu+\alpha-\beta} \mathrm{g}(\mathrm{t}) \mathrm{dt} \\
& +\frac{2}{\pi} \sin \pi(\kappa+\alpha-\beta) \frac{d}{d \rho}\left[\rho^{\kappa-\nu+1} \int_{c}^{\infty} g(t) \phi(\rho, t) d t\right] \text {, say }
\end{aligned}
$$

by using the results of lemmas 2 and 3 , where, 


$$
\phi(\rho, \mathrm{t})=\int_{0}^{\infty} \frac{I_{\nu}(\mathrm{ay})}{K_{\nu}(\mathrm{ay})} K_{\nu-\kappa-1}(\rho \mathrm{y}) K_{\nu-\kappa+\beta-\alpha}(\mathrm{ty}) \mathrm{y}^{\alpha-\beta} \mathrm{dy}
$$

Using the Erdyli-Kober operator $\mathscr{K}_{\eta, \alpha},(3.7)$ can be written as

$$
\begin{gathered}
2^{\alpha-\beta-1} \frac{\mathrm{d}}{\mathrm{d} \rho}\left[\rho^{\kappa-\nu+\beta-\alpha+1} \mathscr{F}_{\frac{1}{2}(\nu-\kappa+\alpha-\beta-1), \beta-\alpha+1}[\mathrm{~g}(\rho)]\right]=\mathrm{F}_{1}(\rho)-\frac{2}{\pi} \sin \pi(\kappa+\alpha-\beta) . \\
\cdot \frac{\mathrm{d}}{\mathrm{d} \rho}\left[\rho^{\kappa-\nu+1} \int_{\mathrm{C}}^{\infty} \mathrm{g}(\mathrm{t}) \phi(\rho, \mathrm{t}) \mathrm{dt}\right] .
\end{gathered}
$$

Or,

$$
\begin{gathered}
\mathscr{F}_{\frac{1}{\frac{1}{2}}(\nu-\kappa+\alpha-\beta+1), \beta-\alpha}[\mathrm{g}(\rho)]=\mathrm{F}_{1}(\rho)+\frac{2}{\pi}^{\beta-\alpha+1} \sin \pi(\kappa+\alpha-\beta) \\
\cdot \rho^{\nu-\kappa+\alpha-\beta} \frac{\mathrm{d}}{\mathrm{d} \rho}\left[\rho^{\kappa-\nu+1} \int_{\mathrm{c}}^{\infty} \mathrm{g}(\mathrm{t}) \phi(\rho, \mathrm{t}) \mathrm{dt}\right] \\
=\mathrm{F}(\rho)+\mathrm{G}(\rho), \text { say. }
\end{gathered}
$$

Therefore, for $0<\alpha-\beta<1$,

$$
\begin{aligned}
\mathrm{g}(\rho)= & \mathscr{T}_{\frac{1}{2}(\nu-\kappa+\alpha-\beta+1), \beta-\alpha}^{-1}[\mathrm{~F}(\rho)+\mathrm{G}(\rho)] \\
= & \mathscr{T}_{\frac{1}{2}(\nu-\kappa+\beta-\alpha+1), \alpha-\beta}[\mathrm{F}(\rho)+\mathrm{G}(\rho)] \\
= & \frac{2}{\Gamma(\alpha-\beta)} \rho^{\nu-\kappa+\beta-\alpha+1} \int_{\rho}^{\infty}\left(\mathrm{u}^{2}-\rho^{2}\right)^{\alpha-\beta-1} \mathrm{u}^{\kappa-\nu+\beta-\alpha}[\mathrm{F}(\mathrm{u})+\mathrm{G}(\mathrm{u})] \mathrm{du} \\
= & -\frac{2^{\beta-\alpha+1}}{\Gamma(\alpha-\beta)} \rho^{\nu-\kappa+\beta-\alpha+1} \int_{\rho}^{\infty}\left(\mathrm{u}^{2}-\rho^{2}\right)^{\alpha-\beta-1} \mathrm{~F}_{1}(\mathrm{u}) \mathrm{du} \\
& \quad+\frac{2^{\beta-\alpha+2}}{\pi \Gamma(\alpha-\beta)} \sin \pi(\kappa+\alpha-\beta) \rho^{\nu-\kappa+\beta-\alpha+1} \int_{\rho}^{\infty}\left(\mathrm{u}^{2}-\rho^{2}\right)^{\alpha-\beta-1} \mathrm{du} . \\
& \quad \cdot \frac{\mathrm{d}}{\mathrm{du}}\left[\mathrm{u}^{\kappa-\nu+1} \int_{\mathrm{c}}^{\infty} \mathrm{g}(\mathrm{t}) \phi(\mathrm{u}, \mathrm{t}) \mathrm{dt}\right] . \\
= & \mathrm{I}_{1}+\mathrm{I}_{2}, \quad \operatorname{say},
\end{aligned}
$$

where $F_{1}$ is defined in equation (3.7). In $I_{1}$, substitute for $F_{1}(u)$ and evaluate the u-integral, using the result of lemma 5 , we obtain

$$
\mathrm{I}_{1}=-2 \rho \int_{0}^{\infty} \frac{\Omega_{\nu-\kappa+\beta-\alpha, \nu}(\xi ; \rho, \mathrm{a})}{\mathrm{J}_{\nu}^{2}(\xi \mathrm{a})+\mathrm{Y}_{\nu}^{2}(\xi \mathrm{a})} \xi^{\alpha-\beta+1} \mathrm{~d} \xi \int_{\mathrm{a}}^{\mathrm{c}} \Re_{\nu-\kappa, \nu}(\xi ; \mathrm{t}, \mathrm{a}) \mathrm{t} \mathrm{f}_{1}(\mathrm{t}) \mathrm{dt},
$$

And $I_{2}$ is similar to $I_{2}$ of (2.13) from whence

$$
I_{2}=-\frac{2}{\pi} \sin \pi(\kappa+\alpha-\beta) \rho \int_{c}^{\infty} g(t) d t \int_{0}^{\infty} \frac{I_{\nu}(\text { ay })}{K_{\nu}(\text { ay })} K_{\nu-\kappa+\beta-\alpha}(\rho y) K_{\nu-\kappa+\beta-\alpha}(t y) y d y
$$

Hence for $0<\alpha-\beta<1, \mathrm{~g}$ satisfies (3.9), a single integral equation of Fredholm type of second kind. 
Note that this equation is identical to the one given in (3.5) for the case $-1<\alpha-\beta<0$. Hence we have a solution for the system (3.1), (3.2) given by

$\psi(\xi)=\xi^{2 \alpha} \int_{\mathrm{a}}^{\mathrm{c}} \Re_{\nu-\kappa, \nu}(\xi ; \rho, \mathrm{a}) \rho \mathrm{f}_{1}(\rho) \mathrm{d} \rho+\xi^{\alpha+\beta} \int_{\mathrm{c}}^{\infty} \Re_{\nu-\kappa+\beta-\alpha, \nu}(\xi ; \mathrm{t}, \mathrm{a}) \mathrm{g}(\mathrm{t}) \mathrm{dt}$

where $g$ satisfies the integral equation (3.5) or (3.9) for $-1<\alpha-\beta<1, \quad \alpha-\beta \neq 0$. Finally combining the solutions (2.15) and (3.9), we obtain a solution for the system (1.1), (1.2).

The analysis throughout this paper is formal and we have not attempted to justify the change of order of integrations. Nevertheless the analysis can be made rigorous by imposing appropriate conditions on the functions involved and abiding by the various restrictions on the parameters, stated at the conclusion of each case considered above.

Next we shall derive some special important cases of the solution of one general system (1.1), (1.2).

\section{SPECIAL CASES}

(a) If we take $\mathrm{k}=0$, the solution corresponds to the one given in [1]. We must point out that the results given in [1] for the case $0<\alpha \leq \frac{1}{2}$ are only valid for $\nu>0$ as mentioned there.

(b) Let $\nu=\kappa=1, \alpha=\frac{1}{2}$ and $\beta=0$. Then the system, from (2.1), (2.2),

$$
\begin{array}{ll}
\mathrm{W}_{0,1}^{-1}\left[\xi^{-1} \psi(\xi) ; \rho\right]=0, & \mathrm{a} \leq \rho \leq \mathrm{c} \\
\mathrm{W}_{0,1}^{-1}[\psi(\xi) ; \rho]=-\mathrm{f}_{2}(\rho), & \mathrm{c}<\rho<\infty
\end{array}
$$

has a solution given by, from (2.11),

$$
\psi(\xi)=\sqrt{\frac{2}{\pi}} \int_{c}^{\infty}\left[\cos (\xi t) Y_{1}(\xi a)-\sin (\xi t) J_{1}(\xi a)\right] t^{-\frac{1}{2}} g(t) d t
$$

where $g$ satisfies the equation,

$$
g(\rho)=\rho^{\frac{1}{2}} \int_{c}^{\infty} t^{-\frac{1}{2}} g(t) d t \int_{0}^{\infty} \frac{I_{1}(\text { ay })}{K_{1}(\text { ay })} e^{-y(\rho+t)} d y-\left[\frac{2 \rho}{\pi}\right]^{\frac{1}{2}} \int_{c}^{\infty} \frac{u}{\sqrt{u^{2}-\rho^{2}}} f_{2}(u) d u .
$$

c) Let $\nu=\kappa=1, \alpha=\frac{1}{2}$ and $\beta=0$. Then the system, from (3.1), (3.2)

$$
\begin{array}{ll}
\mathrm{W}_{0,1}^{-1}\left[\xi^{-1} \psi(\xi) ; \rho\right]=\mathrm{f}_{1}(\rho), & \mathrm{a} \leq \rho \leq \mathrm{c} \\
\mathrm{W}_{0,1}^{-1}[\psi(\xi) ; \rho]=0, & \mathrm{c}<\rho<\infty
\end{array}
$$

has a solution given by, from (3.6),

$$
\psi(\xi)=\xi \int_{\mathrm{a}}^{\mathrm{c}} \boldsymbol{R}_{0,1}(\xi ; \rho, \mathrm{a}) \rho \mathrm{f}_{1}(\rho) \mathrm{d} \rho+\left[\frac{2}{\pi}\right]^{\frac{1}{2}} \int_{0}^{\infty}\left[\cos (\xi \mathrm{t}) \mathrm{Y}_{1}(\xi \mathrm{a})-\sin (\xi \mathrm{t}) \mathrm{J}_{1}(\xi \mathrm{a})\right] \mathrm{t}^{-\frac{1}{2}} \mathrm{~g}(\mathrm{t}) \mathrm{dt}
$$




$$
\begin{aligned}
& \mathbf{g}(\rho)=\rho^{\frac{1}{2}} \int_{\mathrm{c}}^{\infty} \mathrm{t}^{-\frac{1}{2}} \mathrm{~g}(\mathrm{t}) \mathrm{dt} \int_{0}^{\infty} \frac{\mathrm{I}_{1}(\mathrm{ay})}{\mathrm{K}_{1}(\mathrm{ay})} \mathrm{e}^{-\mathrm{y}(\rho+\mathrm{t})} \mathrm{dy} \\
& -\left[\frac{2 \rho}{\pi}\right]^{\frac{1}{2}} \int_{0}^{\infty} \frac{\cos (\xi \rho) \mathrm{Y}_{1}(\xi \mathrm{a})-\sin (\xi \rho) \mathrm{J}_{1}(\xi \mathrm{a})}{\mathrm{J}_{1}^{2}(\xi \mathrm{a})+\mathrm{Y}_{1}^{2}(\xi \mathrm{a})} \xi \mathrm{d} \xi \int_{\mathrm{a}}^{\mathrm{c}} \mathrm{t}_{1}(\mathrm{t}) \boldsymbol{R}_{0,1}(\xi ; \mathrm{t}, \mathrm{a}) \mathrm{dt} .
\end{aligned}
$$

(d) Let $\nu=\kappa=0, \alpha=0$ and $\beta=-\frac{1}{2}$. Then, from (2.1), (2.2), the system of equations

$$
\begin{array}{ll}
\mathrm{W}_{0,0}^{-1}[\psi(\xi) ; \rho]=0, & \mathrm{a} \leq \rho \leq \mathrm{c} \\
\mathrm{W}_{0,0}^{-1}[\xi \psi(\xi) ; \rho]=-\mathrm{f}_{2}(\rho), & \mathrm{c}<\rho<\infty
\end{array}
$$

has a solution, from (2.11),

$$
\psi(\xi)=\frac{2}{\pi \xi} \int_{c}^{\infty}\left[\cos (\xi t) Y_{0}(\xi a)-\sin (\xi t) J_{0}(\xi a)\right] t^{-\frac{1}{2}} g(t) d t
$$

where $\mathrm{g}$ satisfies the equation, from (2.14),

$$
\begin{aligned}
g(\rho) & =\rho^{\frac{1}{2}} \int_{c}^{\infty} t^{-\frac{1}{2}} g(t) d t \int_{0}^{\infty} \frac{I}{K_{0}}\left(\frac{a y}{(a y)} e^{-y(\rho+t)} d y\right. \\
& -\left[\frac{2 \rho}{\pi}\right]^{\frac{1}{2}} \int_{\rho}^{\infty} \frac{u}{\sqrt{u^{2}-\rho^{2}}} f_{2}(u) d u
\end{aligned}
$$

(e) Finally, a purely formal case, when $\nu=\kappa=\frac{1}{2}$ and $\alpha-\beta=\frac{1}{2}$, then

$$
\begin{array}{ll}
\mathrm{w}_{0, \frac{1}{2}}^{-1}\left[\xi^{-2 \alpha} \psi(\xi)\right]=\mathrm{f}_{1}(\rho), & \mathrm{a} \leq \rho \leq \mathrm{c} \\
\mathrm{w}_{0, \frac{1}{2}}^{-1}\left[\xi^{-2 \beta} \psi(\xi)\right]=-\mathrm{f}_{2}(\rho), & \mathrm{c}<\rho<\infty
\end{array}
$$

has a solution given by

$$
\begin{aligned}
& \psi(\xi)=\xi^{\alpha+\beta} \int_{\mathrm{c}}^{\infty} \Re_{-\frac{1}{2, \frac{1}{2}}}(\xi ; \mathrm{t}, \mathrm{a})\left[\mathrm{g}_{1}(\mathrm{t})+\mathrm{g}_{2}(\mathrm{t})\right] \mathrm{dt} \\
&+\xi^{2 \alpha} \int_{\mathrm{a}}^{\mathrm{c}} \Re_{0, \frac{1}{2}}(\xi ; \mathrm{t}, \mathrm{a}) \mathrm{t} \mathrm{f}_{1}(\mathrm{t}) \mathrm{dt}
\end{aligned}
$$

where

$$
\begin{gathered}
g_{1}(t)=\left[\frac{2 t}{\pi}\right]^{\frac{1}{2}} \int_{t}^{\infty} \frac{u}{\sqrt{u^{2}-t^{2}}} f_{2}(u) d u \\
g_{2}(t)=-\pi a t \int_{0}^{\infty} R_{-\frac{1}{2}, \frac{1}{2}}(\xi ; t, a) \xi^{5 / 2} d \xi \int_{a}^{c} u f_{1}(u) R_{0, \frac{1}{2}}(\xi ; u, a) d u
\end{gathered}
$$

with

$$
\begin{gathered}
\mathscr{R}_{-\frac{1}{2}, \frac{1}{2}}(\xi ; \mathrm{t}, \mathrm{a})=-\frac{2}{\pi \xi}(\mathrm{at})^{-\frac{1}{2}} \cos \xi(\mathrm{t}-\mathrm{a}) \\
\mathscr{R}_{0, \frac{1}{2}}(\xi ; \mathrm{t}, \mathrm{a})=-\left[\frac{2}{\pi \xi \mathrm{a}}\right]^{\frac{1}{2}}\left[\cos (\xi \mathrm{a}) \mathrm{J}_{0}(\xi \mathrm{t})+\sin (\xi \mathrm{a}) \mathrm{Y}_{0}(\xi \mathrm{t})\right]
\end{gathered}
$$


The author would like to thank Department de Mathematique, Université de Toulon et du Var, La Garde, France, where this research was carried out. The research is partially supported by a grant from NSERC of Canada.

\section{REFERENCES}

1. SIRIVASTAV, R.P. A pair of dual integral equations involving Bessel functions of the first and the second kind, Proc. Edin. Math. Soc. (2) 14 (1964), 149-158.

2. KRAJEWSKI, J. and OLESIAK, Z.. Associated Weber integral transforms of $\mathrm{W}_{\nu-1, \nu}[;]$ and $\mathrm{W}_{\nu-2, \nu}[;]$ types, Bull. de L'Académe Polonaise des Scs. Vol. $\underline{\mathrm{XXX}}$, No. 7-8, 1982 .

3. NASIM, C. and AGGARWALA, B.D. On some dual integral equations, Ind. J. Pure Math. 15(3), 323-340, March 1984.

4. SNEDDON, I.N. Lecture Notes in Mathematics, No. 457, (Springer), Fractional Calculus (1975).

5. ERDELYI ET AL. Tables of Integral Transforms II, Bateman Manuscript Project, McGraw-Hill, New York (1954). 


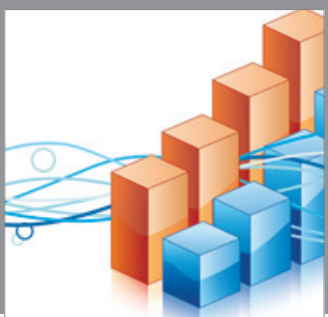

Advances in

Operations Research

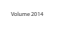

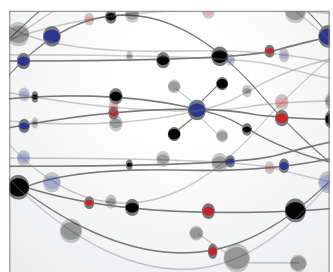

\section{The Scientific} World Journal
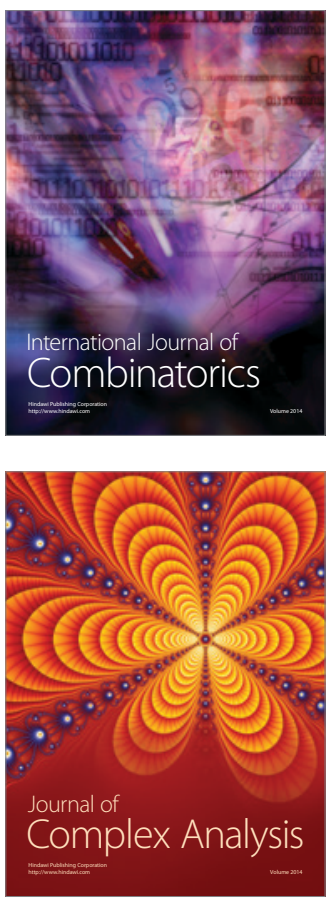

International Journal of

Mathematics and

Mathematical

Sciences
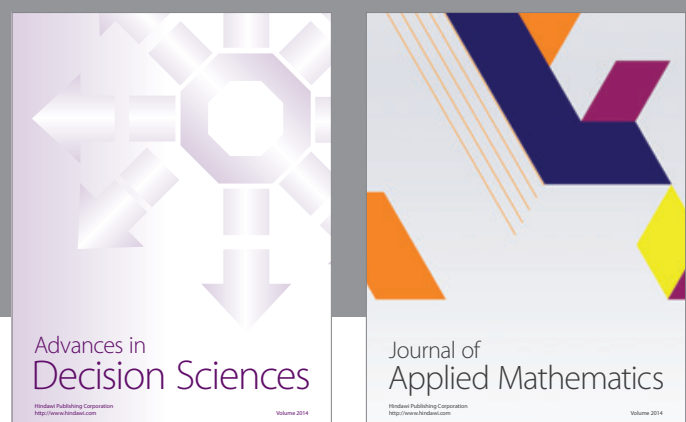

Journal of

Applied Mathematics
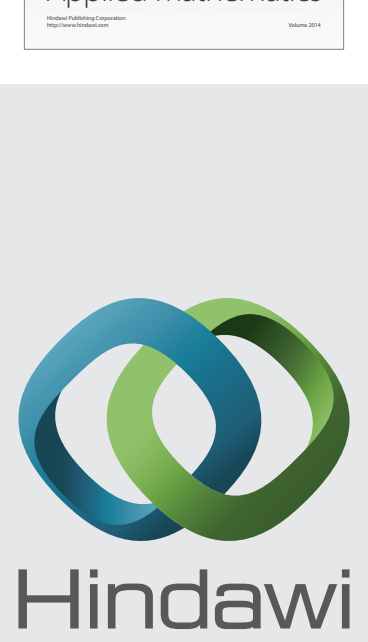

Submit your manuscripts at http://www.hindawi.com
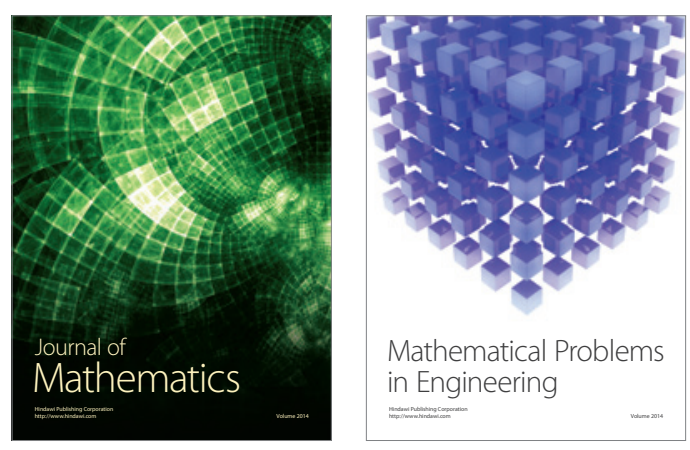

Mathematical Problems in Engineering
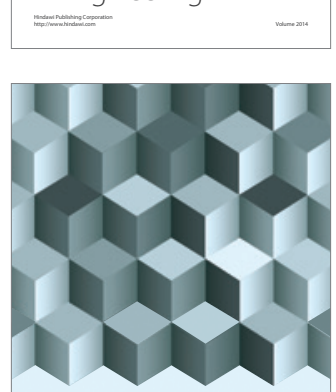

Journal of

Function Spaces
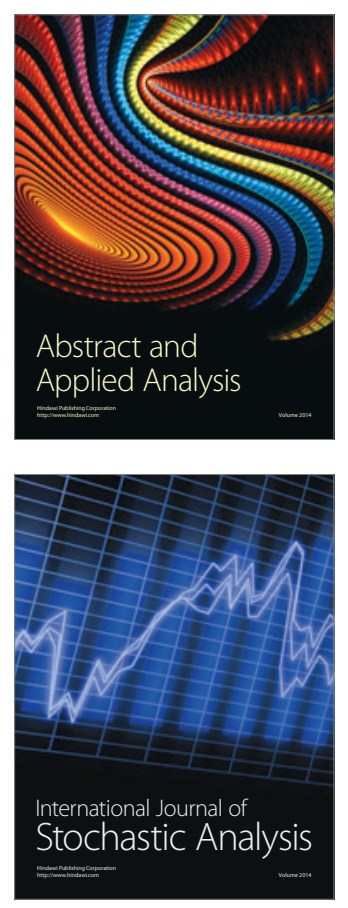

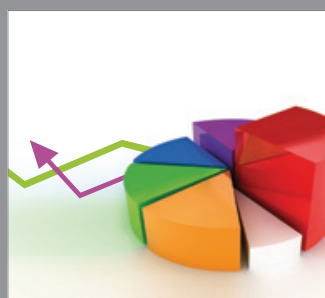

ournal of

Probability and Statistics

Promensencen
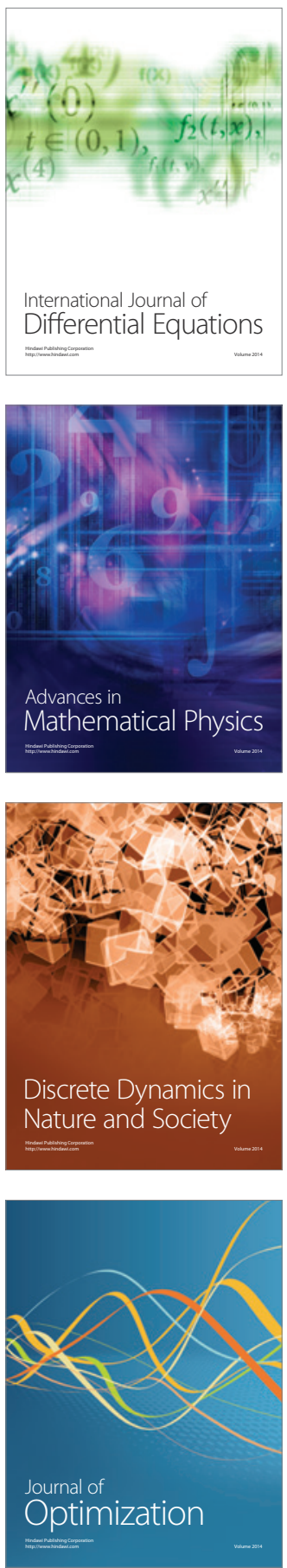